\title{
Spatially embedded socio-technical complex networks
}

\author{
Emanuele Strano, a, Massimiliano Zanin ${ }^{2,3,4}$, Ernesto Estrada ${ }^{5}$, \\ and Fabrizio Lillo ${ }^{6,7,8}$ \\ 1 LaSIG laboratory, Lausanne Institute of Technology (EPFL), Lausanne, Switzerland \\ 2 Innaxis Foundation \& Research Institute, Madrid, Spain \\ 3 Centre for Biomedical Technology, Polytechnic University of Madrid, Spain \\ 4 Faculdade de Ciências e Tecnologia, Universidade Nova de Lisboa, Portugal \\ 5 Department of Mathematics and Statistics, University of Strathclyde, Glasgow, UK \\ 6 Scuola Normale Superiore, Piazza dei Cavalieri 7, 56126 Pisa, Italy \\ 7 Dipartimento di Fisica, viale delle Scienze, 90128 Palermo, Italy \\ 8 Santa Fe Institute, 1399 Hyde Park Road, Santa Fe, NM 87501, USA
}

Complex networks have emerged as a powerful tool for the analysis of complex systems, i.e., systems that are composed of a large number of elements interacting in a non-linear fashion. In the last decade we have contributed to a real Networks Revolution that has percolated many fields of science. Examples range from communication science, genetics and sociology to epidemiology and urban studies. One of the reasons underpinning this success can be found in the natural adaptability of complex networks to different problems. This allows simplification of the system, concentrating attention on patterns of interconnections and essentially enabling all other elements to be neglected. Another great advantage of complex networks was the presence of a mature and solid ground for its development, that is, graph theory and statistical physics.

Further consideration of complex networks might provide us with a more interesting interpretation of the origin of its success. As in many other scientific and non-scientific fields, the deep nature of an object always aspires to re-emerge beyond its latent functions or temporary uses. From time to time, it also transpires that a single idea survives over a significant period of time with different aliases as it moves from field to field, instigating new challenges and gaining its own maturity across generations of scientists and accompanying the evolution of technology. This is the case for Spatially Embedded Socio-Technical Complex Networks, and recently there has been increasing interest in the spatiality of their organisation. Spatiality in network-like objects is certainly not new. Even if extremely important, let us leave aside Euler, the classical Königsberg problems, and the classical results on planar graphs. Let us also assume that the vast and old fields of transportation studies and transportation engineering have been an important step in its application. We might then attempt an easy but useful exercise to determine the origin of the language prior to its mathematical formalisation, and hence the origins of thinking about the world as a collection of connected objects embedded into a space and creating a unique system. The study of ancient cartography provides us with an excellent opportunity for achieving this.

We report here on a very interesting and beautiful example, one of the wonderful maps created by the ancient Persian geographer Ibrahim Al-Istakhri.

\footnotetext{
${ }^{a}$ e-mail: emanuele.strano@epfl.ch
} 


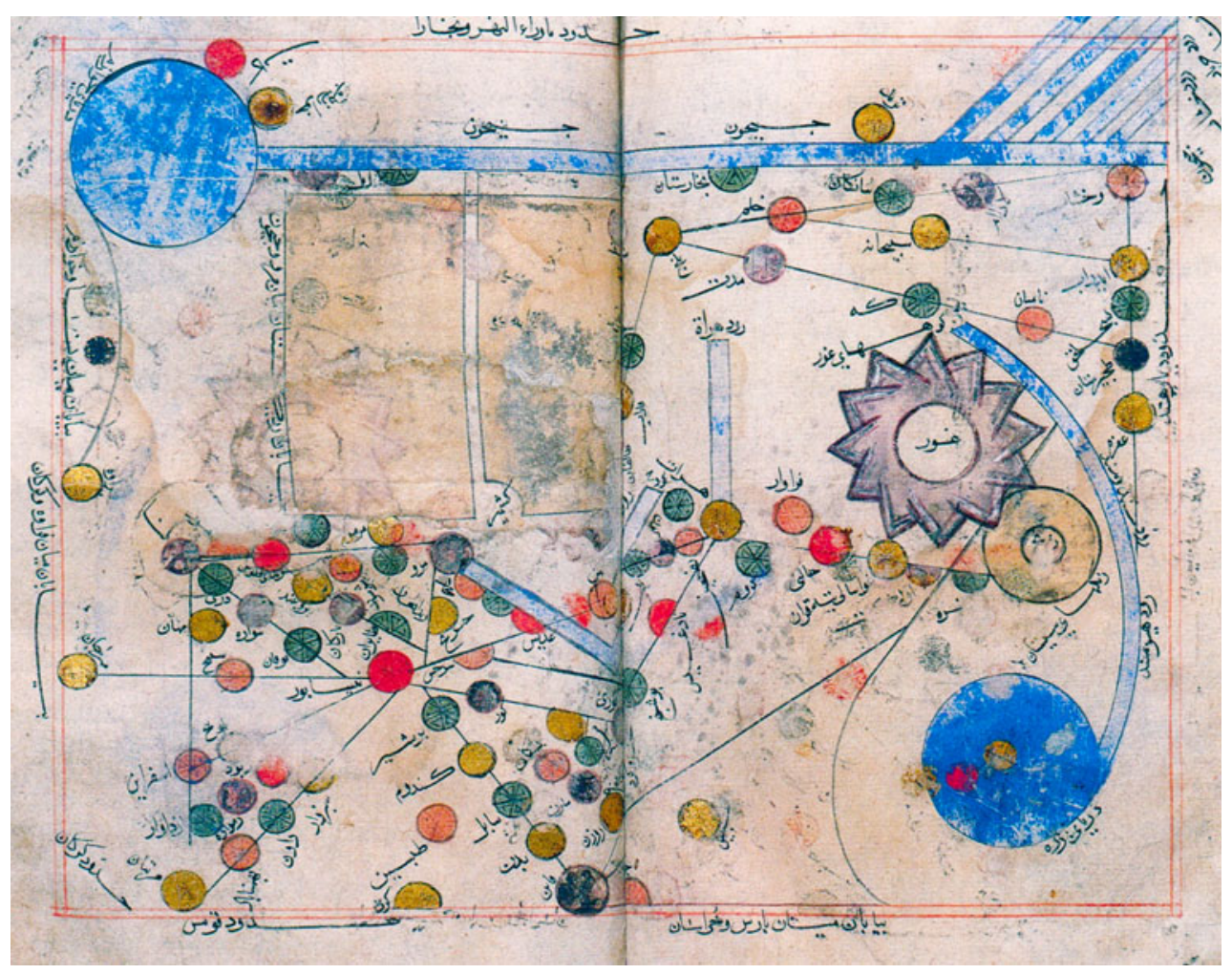

Fig. 1. Map of the Greater Khorasan by the Persian geographer Ibrahim Al-Istakhri, circa 980 AD. Source: Department of Oriental Manuscripts, Bibliothèque National de France.

The map presumably represents the Greater Khorasan, which is a historical region in the northeast of Persia. This map was used by merchants to trace their commercial routes for goods exchange. In it, the coloured circles represent ancient cities. The first version of this map dated from around $980 \mathrm{AD}$, almost eight hundred years before Euler solved the Königsberg problem. Besides interesting considerations related to the interpretation of this map, our primary question is: does this map represent a complex spatial network? We invite the reader to observe it: many geographical entities (cities) are represented by coloured circles, and are connected to each other with black lines representing streets, and with large blue lines representing rivers. This is certainly a procedural map that was used as we would use any contemporary tube map: in other words, this is definitely a network! Nodes are both labeled and coloured, meaning that they provide information regarding their class (this could be a sort of weight but is at least a class). Nodes are also present in different sizes: for instance, those nodes with degree greater than two seem to be larger than the others. There are also half-nodes, which may be bringing additional information related to the nodes' class. Links connecting nodes are divided into two main typologies, i.e., rivers and streets, indicating that this is also a multilayered network. Distances between nodes do not approximate the real distances between geographical entities, which indicates that the links are non-weighted and that the network is fully procedural. Furthermore, streets sometimes intersect both other streets and rivers in a non-planar way; we are therefore facing a three-dimensional embedded graph (?). Links are incident with nodes, but they can be also tangent or transverse, leading to chains and possibly 
fictitious nodes. Finally, we note the presence of unconnected nodes, indicating that the network has different separated components.

Without going further into this game (the colour of the nodes may indicate a kind of centrality, as suggested by the big red node in the lower left-hand corner of the map), we have identified a one thousand year old multilayer, multi-component and potentially weighted network. The fact that this is a map, i.e., a representation of the geography of a place, and that it is not a specific symbolic representation that only a few specialised people were able to understand, means that this network-map was already a part of a mature shared code of representation, and that the origin of this code must be much older than the map itself. We might produce more examples of proto-networks, for instance from the history of cladistic trees or from the history of the evolution of symbolic languages, but we leave the reader to keep enjoining this game. The case we report here aims only to show that spatial problems and network representations have been intrinsically connected for a very long time, and that this is an old marriage, much older than we might typically think. This might also be the reason that this marriage continues to stimulate the scientific community through to the year 2011, when two events were independently organised to deepen this approach: the Evolution of Complex Transportation Networks, a workshop organised at the University of Strathclyde by Emanuele Strano and supported by the Urban Design Studies Unit and the Institute of Complex System at Strathclyde, and the Complexity and the Future of Transportation Systems Satellite Meeting, organised by Fabrizio Lillo and Massimiliano Zanin within the European Conference on Complex Systems 2011 in Wien. This issue of EPJ Special Topics gathers together some of the best contributions presented at both events, as well as from other external contributions.

From urban mobility to worldwide patterns in air transport, this issue presents the reader with an overview of how the movements of people can be described by means of complex networks, and how the evolution of the associated transportation systems through time can be explained. The paper Modeling the Air Transport with Complex Networks: a short review by Massimiliano Zanin and Fabrizio Lillo reviews the most important aspects of air transportation networks; in it, a comprehensive review of works published on this subject is offered to the interested reader, along with considerations of the most promising lines for future researches.

Ranging from large to small scales, some of the most important transportation modes are represented in this issue.

Besides the aforementioned tutorial paper, Azzam et al. analyse the evolution of the worldwide air transport network using a huge data set describing scheduled passenger movements between 1979 and 2007. Among their most important results, they challenge the idea that this network is stationary, demonstrating that it is the result of a combination of accelerated growth and important external constraints (e.g., airline business considerations and global macroeconomics). Cardillo et al. also present a study of air transportation networks that challenges the re-scheduling problem in Europe by means of multiple-layer structure. They define a layer as a given airline and find that, compared with the classical one-layer representation, the structure of the network is changing both quantitatively and qualitatively.

The structure and evolution of the trans-African highway system are studied by Adamatzky et al. by means of an original approach, i.e., the imitation of the development forces by means of the slime mould Physarum polycephalum. When major cities are represented on a surface by sources of nutrients, the slime mould expands in order to connect all of them. Remarkably, the networks created by this organism resemble the real one, shedding light on the self-organised forces defining the growth of human transportation networks, and on the potential of the bio-inspired computing approach. 
At the national level, the mining of large mobility data provides a solid basis for understanding and modeling human mobility. This is the main goal of Pappalardo et al. when they propose the use of unique datasets based on GPS for tracking 10 million journeys undertaken by 150,000 cars in the entirety of Italy.

In a similar fashion, Caschili et al. are proposing a study of community detection in time-varying commuting networks detected over two decades, which is applied to regional planning. Their approach is used to validate the actual number, size and position of political sub-regional partitions in the region of Sardinia, Italy. They conclude that even unconsciously, the recent new Sardinia's partition reflects the real commuters, behaviour.

Barrena et al. propose the use of hypergraphs, i.e., networks composed of multiple interconnected layers, for the study of the topological characteristics of subway networks. Besides the specific results obtained for the networks of Vienna, Los Angeles, Montreal and Moscow, the work extends many essential network metrics to multi-layer scenarios, providing important analytical demonstrations.

The rather peculiar Minneapolis Skyway Network, that connects different buildings in Minneapolis by means of glass-enclosed bridges, is studied by Huang et al. Using historical data ranging from 1962 to 2002, and adopting an agent-based approach, they analyse the forces defining the growth of such networks, and the consequences on the accessibility of different commercial zones.

The evolution of an open source cartographic network, such as the Open Street Map, has been proposed by Corcorain et al. They measure for the first time the topology of a top-down cartographic network, revealing how people map an area and how top-down maps evolve in time. They found that different cases share the same growing processes and therefore that mapping might be governed by universal roles. Their results are indeed linked to the perception of space and navigation strategies in a network-like environment. The reader might link to the navigability indices proposed by Lee et al. for evaluating the local performance of spatial networks; among different interesting findings, remarkably they show that an optimal pedestrian street network should be similar to a triangular grid, such as the street network pattern we are finding in most of the historical centres.

Transportation may not simply refer to people: in modern society communication networks are essential due to their large impact on the economy and on our way of life. Chen et al. propose a model for explaining the structure (including the spatial structure) of access nodes within the Internet infrastructure.

We would love to send a copy of this issue to Ibrahim Al-Istakhri for his comments and to illustrate to him how much development networks have progressed since his pioneering efforts. The study of complex networks has moved from qualitative analysis to a quantitative science, in which models and measures are used to explain not-sotrivial facts hidden within the structural patterns of connectivity. Despite many of the advances seen today, there is still room for the development of new qualitative and quantitative tools for analysing complex transportation networks in a way that more extensive and reliable information is extracted from them.

We are extremely grateful to the European Physical Journal Special Topics for having hosted this issue and in particular to Christian Caron and Sabine Lehr. We furthermore express our sincere gratitude to the authors of the different manuscripts, and to all of our colleagues who have assisted us in the reviewing process and preparation of this special issue. Emanuele Strano is particularly grateful to François Golay, Marc Barthèlemy and Sergio Porta for their constant support, to Andrea Perna, Gianluca Oppo and Paola Pasino for their help in the organisation of the workshop Evolution of Complex Transportation Networks, and Luigi Farrauto for providing the map of Greater Khorasan. 